\title{
The telomere length of gastric mucosal samples and peripheral blood lymphocytes in patients who have undergone Billroth II distal gastrectomy
}

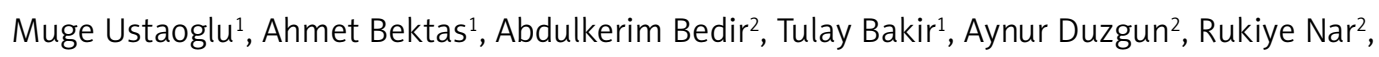
Ozgur Ecemis ${ }^{1}$, Rahmi Aslan ${ }^{1}$

1Department of Gastroenterology, Ondokuz Mayis University, Faculty of Medicine, Samsun, Turkey

${ }^{2}$ Department of Medical Biochemistry, Ondokuz Mayis University, Faculty of Medicine, Samsun, Turkey

Submitted: 10 July 2017

Accepted: 21 December 2017

Arch Med Sci 2020; 16 (3): 577-583

DOI: https://doi.org/10.5114/aoms.2020.94656

Copyright $\odot 2020$ Termedia \& Banach

\section{Abstract}

Introduction: Telomeres play an important role in maintaining chromosomal integrity. Functional loss of telomeres increases the risk of cancer by causing genomic instability. Telomere length abnormalities have been reported in several precancerous lesions. There is no study that evaluates telomere length in Billroth II distal gastrectomy, which is known as a risk factor for gastric stump carcinogenesis, in the literature. The aim of this study was to assess the relationship between the telomere length of residual gastric mucosal samples, peripheral blood lymphocytes, and other clinicopathological parameters of patients who had undergone Billroth II distal gastrectomy.

Material and methods: There were two groups: a control group $(n=15)$ and a patient group $(n=15)$. In all cases, upper gastrointestinal endoscopy was performed, and biopsies were taken during endoscopy. Telomere lengths were measured by qRT-PCR.

Results: It was observed that the lengths of the telomeres were shortened as the time of postoperative period increased in the patient group ( $r=$ $-0.126)(p>0.05)$. Also, the lengths of the telomeres were shortened in chronic inflammation, neutrophil activity, glandular atrophy, and intestinal metaplasia.

Conclusions: The telomere length was shortened as the time of postoperative period increased in the patient group. The telomeres were also shorter in chronic inflammation, neutrophil activity, intestinal metaplasia, and glandular atrophy, in all of the study groups. Telomere length abnormalities in gastric stump carcinogenesis process may be a guide for early diagnosis and treatment.

Key words: Billroth II gastrectomy, telomere length, quantitative polymerase chain reaction.

\section{Introduction}

In recent years, a prominent decrease has been observed in partial gastrectomy applications, mainly due to enhanced comprehension of pathophysiology of peptic ulcer disease and development of efficient medical treatment regimes. It is well known that development of cancer is increased in remnant stomach among patients in whom partial

\author{
Corresponding author: \\ Muge Ustaoglu \\ Department \\ of Gastroenterology \\ Faculty of Medicine \\ Ondokuz Mayis University \\ Samsun, Turkey \\ Phone: +90 5322845719 \\ E-mail: ustaoglu.md@gmail. \\ com
}


gastrectomy was performed. This risk is greater in distal gastrectomies with Billroth II as compared to Billroth I [1]. However, the cause of the increase in development of cancer in remnant stomach is not fully understood yet. A number of hypotheses like decrease in acid and gastrin secretion, duodenogastric reflux (DGR), Helicobacter pylori ( $H$. pylori), and bacterial overgrowth have been associated with this issue, but the factor which is most commonly associated is DGR [1, 2].

Structures composed of repetitive deoxyribonucleic acid (DNA) chains, located at the end of chromosomes are called telomeres. Under normal conditions, terminal regions of chromosomes are protected by telomeres. Telomeres that are shortened to critical levels lose their functions. Peripheral blood leukocyte telomere length attrition is associated with aging and age-related diseases such as diabetes mellitus, coronary artery disease, hypertension, and all-cause mortality [3]. Loss of function in telomeres causes genomic instability [4]. Telomerase is an enzyme that prevents shortening of telomeres. Under normal conditions, a limited number of divisions occur in somatic cells and no telomerase activity is seen; but in cells that are active in terms of mitosis, like skin, peripheral blood, and endometrium, low levels of telomerase activity have been found [5]. It has been reported that in telomerase knockout mice, the incidence of cancer has increased. A telomerase activity of $85 \%$ was shown in cancer cells $[6,7]$.

Carcinogenesis is a multi-step process. Changes in telomere length are the most frequent and earliest changes seen during this process [6]. A number of methods have been developed to measure the length of telomere. Quantitative polymerase

Table I. Inclusion criteria

18 years of age and older

Patients with Billroth II distal gastrectomy for peptic ulcer and diagnosed with alkaline reflux gastritis (ARG) in upper GIS endoscopy

Table II. Exclusion and termination criteria

Under 18 years of age

Chronic systemic diseases (diabetes mellitus, hypertension, coronary artery disease, chronic obstructive pulmonary disease, cerebrovascular disease, chronic renal failure)

During the previous 4 weeks, use of antibiotics, non-steroidal anti-inflammatory drugs, proton pump inhibitors, $\mathrm{H} 2$ receptor antagonists, sucralate, cholestyramine, or prokinetic agents

Cancer

Smoking

Patients without informed consent chain reaction ( $q-P C R)$ is a rapid and simple technique to measure telomere length [8].

In the literature, telomere length abnormalities have been reported in early premalignant lesions and predisposing factors for development of cancer. It is well known that ARG is a risk factor for gastric remnant cancer. So, the telomere length abnormalities in the peripheral blood lymphocytes and in gastric mucosa of the patients with Billroth grade II distal gastrectomy for peptic ulcer were calculated, and the relationship between telomere length and other clinicopathological parameters was investigated. In the literature, we were unable to point out any study investigating telomere length in a patient population with Billroth grade II distal gastrectomy for peptic ulcer. In the process of gastric remnant carcinogenesis, abnormalities in length of telomere may be used as a guide in early diagnosis and treatment of gastric remnant cancers.

\section{Material and methods}

\section{Patient selection}

This study was conducted in the Gastroenterology Department of Ondokuz Mayis University Medical School during a period of eight months. A total of 30 volunteers: 15 patients and 15 controls, were enrolled in this study. Inclusion and exclusion criteria are indicated in Tables I and II, respectively.

The control group comprised patients comparative to the patient group in terms of age and gender, with no history of gastric surgery, for whom upper gastrointestinal (GI) endoscopy had been planned due to dyspeptic symptoms with normal endoscopic findings. Exclusion and termination criteria for the control group are the same as for the patient group; in addition to the conditions stated above, patients with primary DGR and patients subjected to cholecystectomy or endoscopic biliary sphincterotomy were also excluded.

Upper Gl endoscopy was performed by the same physician. For histopathological examination, samples were evaluated according to Sydney classification [9]. Endoscopic findings of enterogastric bile reflux, retaining of bile in the gastric remnant pouch, and hyperaemic oedematous appearance of gastric mucosa were regarded as a diagnosis of ARG.

\section{Obtaining lymphocytes and tissue samples}

During upper Gl endoscopy, biopsy specimens were taken from the gastric stump within $1 \mathrm{~cm}$ of the anastomosis in the patient group and from the gastric body in the control group; biopsies were obtained from four quadrants, 6-8 in total, with standard size biopsy forceps, and samples were sent to a laboratory in HBSS solutions. All tissue samples were kept at $-70^{\circ} \mathrm{C}$ until examination. 
Before upper Gl endoscopy, $4.5 \mathrm{ml}$ blood was obtained from the patients in tubes containing EDTA. For isolation of lymphocytes, $1.5 \mathrm{ml}$ blood was added over $1 \mathrm{ml}$ Histopaque-1077,forming a layer and centrifuged for 20 min at $1500 \times \mathrm{g}$ at $4^{\circ} \mathrm{C}$. After centrifuge the supernatant was transferred to another tube and $1 \mathrm{ml}$ HBSS was added; it was centrifuged again at $400 \times \mathrm{g}$ at $4^{\circ} \mathrm{C}$. Supernatant was eliminated, $400 \mu \mathrm{l} \mathrm{HBSS}, 50 \mu \mathrm{l}$ autologus plasma, and $50 \mu \mathrm{l}$ DMSO were added, and cells were resuspended. Samples were kept at $-70^{\circ} \mathrm{C}$ until examination.

\section{Application of real-time quantitative PCR}

Total DNA was extracted from tissue specimens and lymphocytes using the High Pure PCR Template Preparation Kit (Roche Diagnostics). All DNA samples were adjusted to $50-100 \mathrm{pg} / \mu \mathrm{l}$ and $40-50 \mathrm{pg} / \mu \mathrm{l}$ for $\beta$-globin and telomere PCR, respectively. Standards were generated from the reference human genomic DNA sample (LightCycler-Control Kit DNA, Roche Diagnostics) serially diluted two-fold per dilution to produce seven concentration of DNA ranging from 46.9 to 3000 pg/ $\mu \mathrm{l}$ for the $\beta$-globin PCR and to produce six concentrations of DNA ranging from 65.6 to $2100 \mathrm{pg} / \mu \mathrm{l}$ for telomere PCR.

The $\beta$-globin and telomere copy numbers in the samples were determined by absolute standard curve method using a LightCycler (Roche Diagnostics, Germany). To normalize the relative telomere length by real-time qPCR, the $\beta$-globin gene was used as a single-copy gene. Telomere lengths were measured using a qPCR method developed by Cawthon [10], with some modifications. Cawthon's forward and reverse telomere primer sequences were 5'-cggtttgtttgggttt-gggtttgggtttgggtttgggtt-3' and 5'-ggcttgccttacccttacccttacccttacccttaccct-3', respectively. PCR amplification was also performed for 3.9 and 10.2 kilobase pairs long human telomere standards (TeloTAGGG telomere length assay kit, Roche).

The relative telomere length was determined using the $\Delta \Delta \mathrm{Ct}$ method. All samples were normalised by the ratio of telomere PCR product to $\beta$-globin PCR product (T/S ratio). This is formulated as

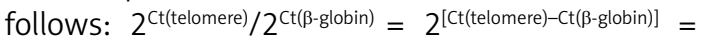
$2^{-\Delta C t}$. Then, the relative T/S ratio was calculated following formula $2^{\Delta \mathrm{ct}(\text { sample) }} / 2^{\Delta \mathrm{ct} \text { (calibrator) }}=2^{\Delta \mathrm{ct} \text { (sample) }}$ $-\Delta c t$ (calibrator) $=2^{-\Delta \Delta c t}$. We used 3.9 kilobase pairs long telomere standard as the calibrator in our study. Hence, the telomere lengths of the samples are found as kilobase pairs.

\section{Statistical analysis}

All data were evaluated by SPSS 13.0 package program. The $\chi^{2}$ test, Fisher's exact test, Mann
Whitney $U$ test, and Spearman correlation test were used, with the level of significance set at $p<0.05$.

\section{Results}

A total of 30 cases: 15 patients and 15 as the control group, were enrolled in the study. The mean age in the patient group was $61.5 \pm 6.5$ years (49-75), and $61.5 \pm 6.4$ years (49-75) in the control group $(p>0.05)$. All patients in both groups were men.

Mean telomere length in gastric mucosa samples was $14972.0 \pm 8122.9$ base pairs (bp) in the patient group and $11266.0 \pm 3466.4 \mathrm{bp}$ in the control group, and telomeres were found to be longer in the patient group as compared to the controls $(p>0.05)$. Mean telomere length in peripheral blood lymphocytes was $10728.7 \pm 6595.0 \mathrm{bp}$ and $13238.0 \pm 5051.5 \mathrm{bp}$ in the patient and the control groups, respectively.

No difference was found in both groups in terms of the presence of histopathological diagnosis, according to Sydney classification.

In all mucosa samples regardless of groups, correlation between histopathological diagnosis according to Sydney classification and telomere length was evaluated. In all different histopathological diagnoses, a shortening in telomere length was observed.

In the study groups, correlation between histopathological diagnosis according to Sydney classification and telomere lengths in gastric mucosa samples were evaluated (Table III).

In the patient group, mean duration since surgical operation was $27.7 \pm 6.6$ year ( $15-45$ years). A negative correlation was found between the time since operation and telomere lengths in gastric mucosa samples, but it was statistically insignificant $(r=-0.126)(p>0.05)$ (Figure 1).

The correlation between the age of the patients and telomere length of peripheral blood lymphocytes and of gastric mucosa samples was evaluated for the patient and the control groups. Negative correlation was found between age and telomere length in lymphocytes from peripheral blood and in gastric mucosa samples in the control group; in the patient group, negative correlation was determined between age and telomere lengths in lymphocytes, while there was positive correlation between age and telomere lengths in gastric mucosa samples $(p>0.05)$ (Figure 2$)$.

\section{Discussion}

Nowadays, surgical treatment is rarely needed in peptic ulcer disease, mainly due to enhanced comprehension of disease pathophysiology and development of powerful drugs for acid inhibition. 


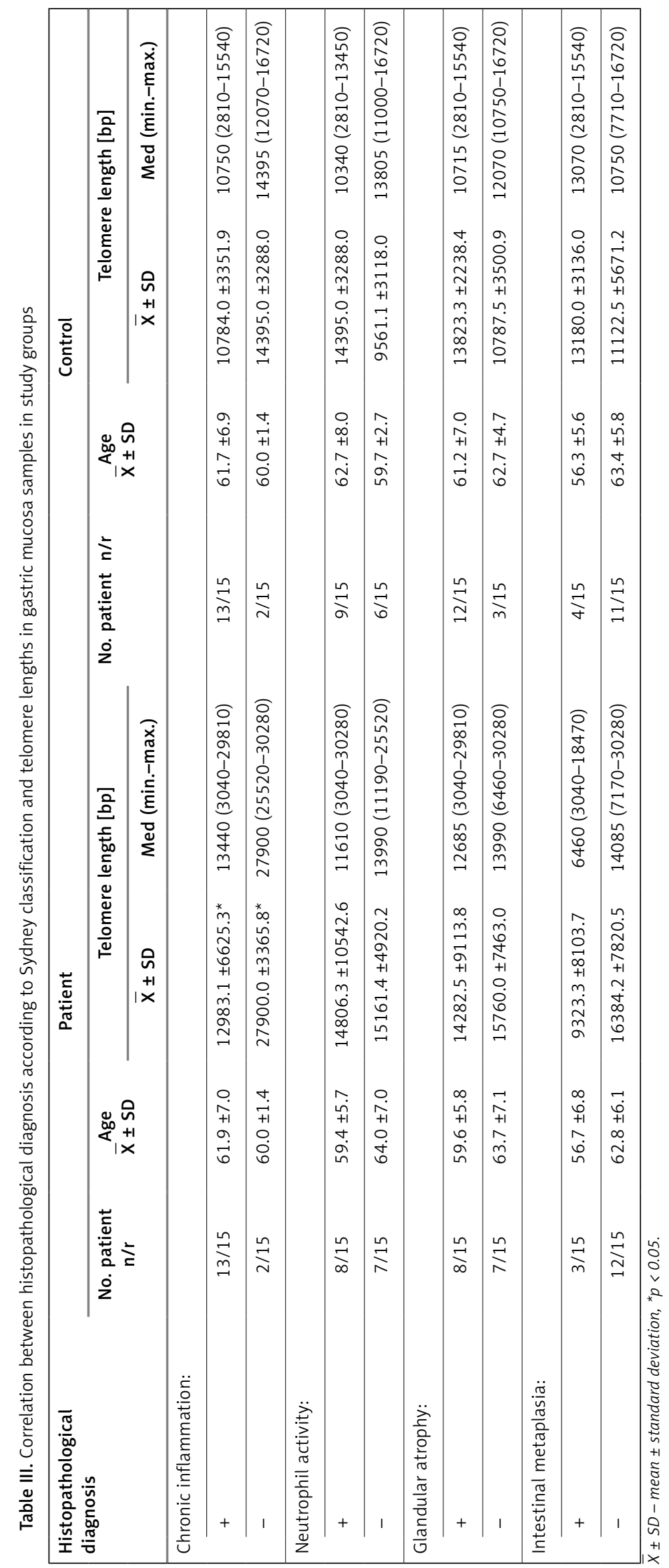


Therefore, acute and especially chronic complications that were previously reported in association with peptic ulcer surgery have decreased considerably. One of the important complications during the chronic stage is the development of cancer in remnant gastric tissue. The risk of cancer development is higher in patients with Billroth grade II as compared to Billroth grade I distal gastrectomy [1]. The aetiology of cancer in remnant stomach after peptic ulcer surgery is not fully understood yet. The main factors indicated in the aetiology are decrease in acid and gastrin secretion, DGR, H. pylori, and bacterial overgrowth. Duodenogastric reflux is the factor that is most frequently attributed to the development of cancer in remnant gastric tissue [1, 2].

Carcinogenesis is a multi-step process, and a number of structural changes are seen during this period. One of the earliest chromosomal changes are abnormalities in telomere length [6]. Telomeres are nucleoprotein structures located in terminal regions of eukaryotic chromosomes. In somatic cells of human beings, telomeres are hexanucleotide DNA sequences, repeating 10002000 times (TTAGGG) [11]. Telomeres play a role in protection of chromosome integrity by stabilising terminal regions of chromosomes. With every cell division, telomeres are somewhat shortened due to end of replication problems. As division of cells increases, shortening of telomeres is also increased in parallel. Telomeres shortened to critical levels lose their functions and hence the cell is not able to go through any further division. Loss of function in telomeres is an important factor in the development of chromosome abnormalities. Progressive shortening of telomeres in advanced age leads to an increase in cancer risk by causing genomic instability [6].

In order to maintain cell division in normal cells with high proliferation ability and in cancer cells, the length of the telomere should be preserved. The length of the telomere is maintained by telomerase and by alternative lengthening of telomeres (ALT), which is currently suggested to be active only in cancer cells and cancer cell cultures. In a number of studies, telomerase enzyme activity was found in peripheral blood, cord blood, bone marrow leucocytes, basal layer of epidermis, endometrium, and chorionic villi [5]. Telomerase enzyme activity was also determined in approximately $85 \%$ of cancer cells. It was confirmed that telomerase activation is required for immortality of the cell and hence is an essential step in carcinogenesis [12]. Studies investigating the arrangement of telomerase activity in gastric cancer assisted in comprehending the molecular mechanisms that play a role in the transition process from atrophic gastritis to gastric cancer.

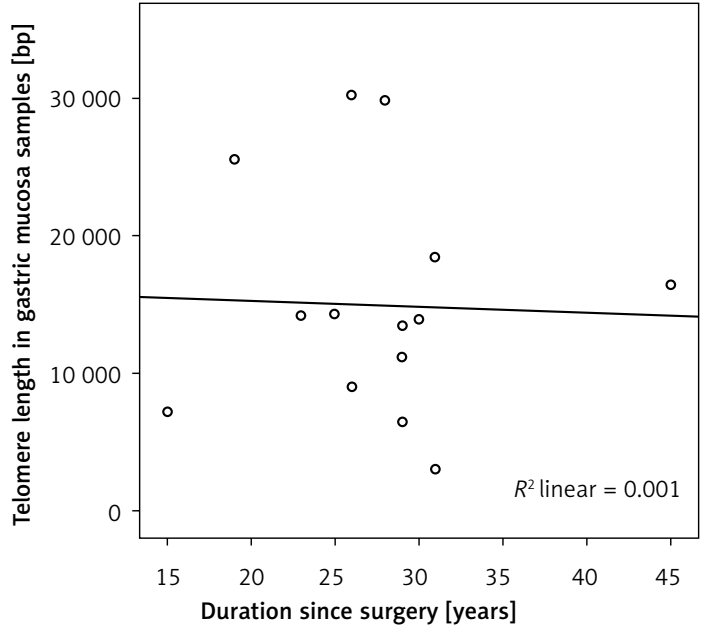

Figure 1. Correlation between duration since operation and telomere length in gastric mucosa samples in the patient group

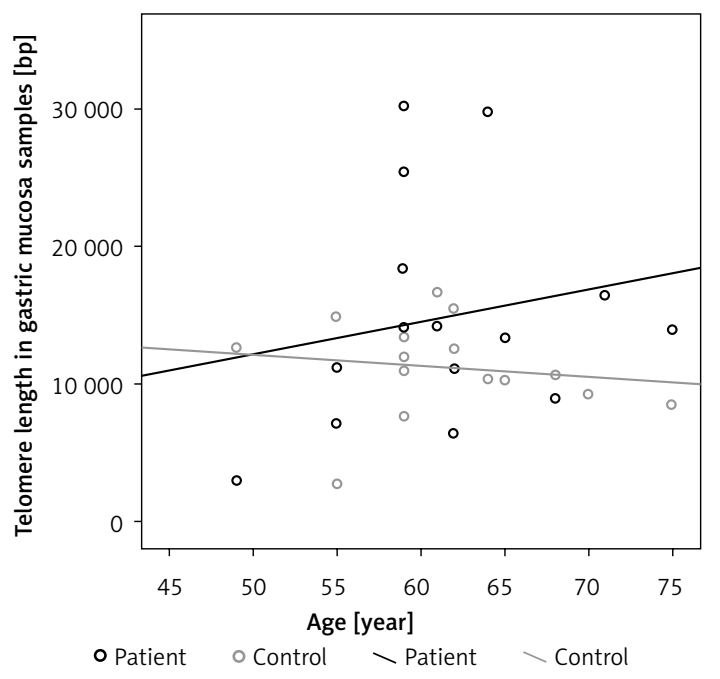

Figure 2. Correlation between age and telomere lengths in gastric mucosa samples in study groups

Discussions about the stage of the carcinogenesis process during which telomerase activity plays a more important role are still valid [13]. In a study conducted by Yang et al., the length of telomere was found to be shortened in chronic atrophic gastritis patients in parallel with progression of the disease [13]. In different studies it was reported that telomeres were shorter in patients with chronic inflammation and intestinal metaplasia $[14,15]$. In our study, we determined histopathological changes with a potential to progress to gastric cancer in the patient group. In both groups, telomere length was shortened in gastric mucosa in the presence of inflammation, glandular atrophy, and intestinal metaplasia. These findings may reflect early events of the carcinogenetic process. However, due to the low number of cases in the patient and the control groups, reliable statistical comparisons could not be performed. 
Most epithelial cancers develop on precursor lesions, which are also known as intraepithelial neoplasia. In some studies, the discovery of genetic instability in intraepithelial tumours suggested that genetic changes develop early in malignant transformation. Meeker et al. indicated that in situations where telomere function disorder is a factor in genetic instability, telomere functions may also be destroyed in early premalignant lesions [6]. In about $90 \%$ of premalignant prostate and pancreas lesions, shortening of telomeres was observed [16-18]. In a study conducted in 35 intraepithelial neoplasia in the oral cavity, oesophagus, colon, bladder, and cervix, the most frequent telomere length abnormality was reported to be shortening of telomere [6]. It is well known that ulcerative colitis, which is a chronic inflammatory intestinal disease, is a predisposing factor for the development of colorectal cancer. In a study carried out among patients with ulcerative colitis, the length of telomere was reported to be shortened in colon epithelial cells of patients, as compared to the control group [19]. In gastroesophageal reflux disease, telomere length in the distal oesophagus was found to be shortened as compared to the control group ( $p<0.05)$, and in Barrett oesophagus, telomere was found to be shortened further, but it was reported that this was not statistically significant $(p>0.05)$ [20]. Shortening of telomere was not determined in the proximal oesophagus, which is less affected by reflux. In another study, the telomere length was shorter in patients with Barrett oesophagus than in those with gastroesophageal reflux disease [21].

In a study conducted by Yang et al., telomere length was evaluated in premalignant gastric lesions by southern blot (SB) method, and telomeres were observed to be gradually shortened, starting from normal mucosa and progressing to atrophic gastritis, intestinal metaplasia, and dysplasia [13]. In our study, comparison of the patient and the control groups revealed an increase of telomere length in gastric mucosa samples $(p>0.05)$ and a decrease of telomere length in lymphocytes $(p>$ 0.05). A decrease of telomere length in peripheral blood lymphocytes of the patient group may present as an early finding of carcinogenesis.

In studies evaluating the correlation between telomere length and age, it was reported that telomeres are shortened with age, but this correlation disappears in advanced ages [22, 23]. In our study, we determined a negative correlation between age and telomere length in peripheral blood lymphocytes $(r=-0.108)$ and a positive correlation between age and telomere length in gastric mucosa in the patient group $(r=0.189)$, but these correlations were found to be statistically insignificant $(p>0.05)$. On the other hand, in the control group, negative correlation was found between age and telomere length in peripheral blood lymphocytes $(r=-0.345)$ and between age and telomere length in gastric mucosa $(r=-0.280)$, and these correlations were also found to be statistically insignificant $(p>0.05)$. In the patient group, unlike the control group, the presence of positive correlation between age and telomere length in gastric mucosa samples, and negativity of this correlation in peripheral blood lymphocytes, suggest that a different factor affecting telomere length may be present in gastric mucosa samples. Since telomerase activity was not evaluated in our study, we have no information whether this enzyme is the factor that increases telomere length or not.

In our study, the increase in mean telomere lengths in gastric mucosa of the patients as compared to the control group may be related to various factors. One of these causes may be telomerase activation, which develops in the early stages of carcinogenesis. The increase in telomere length may be due to normal ALT-like activity [24]. Also, it was reported that terminal-terminal chromosome fusions seen in several tumours may also lead to an increase in telomere length [25]. However, in our study, neither telomerase activity nor structural changes in chromosomes were evaluated; therefore, we are unable to comment on this issue.

Telomere length also varies in different cells. In a study evaluating the association between the telomere length and complete blood count, a negative relationship between telomere length and monocyte count, and a positive relationship with basophil ratio was found [26]. This variance is also valid for different cells of the same organ. Considering that gastric mucosa is composed of various cells, such as epithelial cells, fibroblasts, inflammatory cells, and smooth muscle cells, it should be noted that the telomere lengths of these cells may be different. It was reported that in the stomach, intramucosal lymphocytes possess the longest telomeres, as compared to other mucosa cells [27]. In patients with Billroth II distal gastrectomy, chronic inflammatory cells, and especially lymphocytes, increase in gastric mucosa [28]. Thus, telomeres in gastric mucosa samples may have been found to be longer in the patient group, as compared to the control group. In our study, it was not possible to form a definite opinion on this issue because gastric mucosa cells were not differentiated.

In our study, we measured telomere length in peripheral blood lymphocytes and in histological changes with a potential to progress to gastric cancer in gastric mucosa, and we found telomere length shortening in gastric mucosa. When compared to the control group, an increase in mean 
telomere length in gastric mucosa was found, whereas a decrease in mean telomere length in blood lymphocytes was found in the patient group. Although these results are not statistically significant, which may be due to the low number of patients, we find this result interesting. We think that this may be related to some changes affecting the telomere length in the early stages of carcinogenesis in gastric mucosa, such as telomerase activation, ALT-like activity, or terminal chromosome fusions. But in our study, none of these early stage carcinogenesis changes were evaluated, so it is a limitation of our study. In view of these results, we believe that telomere length and telomerase activity in the early stages of gastric remnant carcinogenesis may be regarded as beneficial molecular indicators in early diagnosis and treatment of this disease. We suggest that further studies conducted in large patient populations will be appropriate to claim that determination of telomere length abnormalities are beneficial in the early stages of gastric remnant carcinogenesis.

\section{Acknowledgments}

The study was conducted at the Department of Gastroenterology, Ondokuz Mayis University, Faculty of Medicine, Samsun, Turkey.

\section{Conflict of interest}

The authors declare no conflict of interest.

\section{References}

1. Miwa K, Hattori T, Miyazaki I. Duodenogastric reflux and foregut carcinogenesis. Cancer 1995; 75: 1426-32.

2. Sinning C, Schaefer N, Standop J, et al. Gastric stump carcinoma - epidemiology and current concepts in pathogenesis and treatment. Eur J Surg Oncol 2007; 33: 133-9.

3. Mazidi M, Michos ED, Banach M. The association of telomere length and serum 25-hydroxyvitamin $D$ levels in US adults: the National Health and Nutrition Examination Survey. Arch Med Sci 2017; 13: 61-5.

4. Callén E, Surrallés J. Telomere dysfunction in genome instability syndromes. Mutat Res 2004; 567: 85-104.

5. Bachor C, Bachor OA, Boukamp P. Telomerase is active in normal gastrointestinal mucosa and not up-regulated in precancerous lesions. J Cancer Res Clin Oncol 1999; 125: 453-60.

6. Meeker AK, Hicks JL, lacobuzio-Donahue CA, et al. Telomere length abnormalities occur early in the initiation of epithelial carcinogenesis. Clin Cancer Res 2004; 10: 3317-26.

7. Shay JW, Bacchetti S. A survey of telomerase activity in human cancer. Eur J Cancer 1997; 33: 787-91.

8. Lin KW, Yan J. The telomere length dynamic and methods of its assessment. J Cell Mol Med 2005; 9: 977-89.

9. Dixon MF, Genta RM, Yardley JH, et al. Classification and grading of gastritis. The updated Sydney System. International Workshop on the Histopathology of Gastritis, Houston 1994. Am J Surg Pathol 1996; 20: 1161-81.
10. Cawthon RM. Telomere measurement by quantitative PCR. Nucleic Acids Res 2002; 30: e47.

11. Moyzis RK, Buckingham JM, Cram LS, et al. A highly conserved repetitive DNA sequence, (TTAGGG)n, present at the telomeres of human chromosomes. Proc Natl Acad Sci USA 1988; 85: 6622-6.

12. Kim NW, Piatyszek MA, Prowse KR, et al. Specific association of human telomerase activity with immortal cells and cancer. Science 1994; 266: 2011-5.

13. Yang SM, Fang DC, Luo YH, et al. Alterations of telomerase activity and terminal restriction fragment in gastric cancer and its premalignant lesions. J Gastroenterol Hepatol 2001; 16: 876-82.

14. Maruyama Y, Hanai H, Kaneko E. Telomere length and telomerase activity in intestinal metaplasia, adenoma and well differentiated adenocarcinoma of the stomach. Nippon Rinsho 1998; 56: 1186-9.

15. Tahara T, Shibata T, Kawamura T, et al. Telomere length shortening in gastric mucosa is a field effect associated with increased risk of gastric cancer. Virchows Arch 2016; 469: 19-24.

16. Meeker AK, Hicks JL, Platz EA, et al. Telomere shortening is an early somatic DNA alteration in human prostate tumorigenesis. Cancer Res 2002; 62: 6405-9.

17. Vukovic B, Park PC, Al-Maghrabi J, et al. Evidence of multifocality of telomere erosion in high-grade prostatic intraepithelial neoplasia (HPIN) and concurrent carcinoma. Oncogene 2003; 22: 1978-87.

18. van Heek NT, Meeker AK, Kern SE, et al. Telomere shortening is nearly universal in pancreatic intraepithelial neoplasia. Am J Pathol 2002; 161: 1541-7.

19. O'Sullivan JN, Bronner MP, Brentnall TA, et al. Chromosomal instability in ulcerative colitis is related to telomere shortening. Nat Genet 2002; 32: 280-4.

20. Souza RF, Lunsford T, Ramirez RD, et al. GERD is associated with shortened telomeres in the squamous epithelium of the distal esophagus. Am J Physiol Gastrointest Liver Physiol 2007; 293: G19-24.

21. Wennerström EC, Risques RA, Prunkard D, et al. Leukocyte telomere length in relation to the risk of Barrett's esophagus and esophageal adenocarcinoma. Cancer Medicine 2016; 5: 2657-65.

22. O'Sullivan J, Risques RA, Mandelson MT, et al. Telomere length in the colon declines with age: a relation to colorectal cancer? Cancer Epidemiol Biomarkers Prev 2006; 15: 573-7.

23. Woo J, Suen EW, Leung JC, et al. Older men with higher self-rated socioeconomic status have shorter telomeres. Age Ageing 2009; 38: 553-8.

24. Reddel RR. Alternative lengthening of telomeres, telomerase, and cancer. Cancer Lett 2003; 194: 155-62.

25. Lindsey J, McGill NI, Lindsey LA, et al. In vivo loss of telomeric repeats with age in humans. Mutat Res 1991; 256: 45-8.

26. Mazidi M, Penson P, Banach M. Association between telomere length and complete blood count in US adults. Arch Med Sci 2017; 13: 601-5.

27. Kameshima H, Yagihashi A, Yajima T, et al. Helicobacter pylori infection: augmentation of telomerase activity in cancer and noncancerous tissues. World J Surg 2000; 24: 1243-9.

28. Burden WR, Hodges RP, Hsu M, et al. Alkaline reflux gastritis. Surg Clin North Am 1991; 71: 33-44. 